\title{
Fighting tuberculosis in the EU/EEA: towards the new European Union standards on tuberculosis care
}

\author{
Giovanni Sotgiu (ib), Netta Beer ${ }^{2}$, Stefano Aliberti ${ }^{3}$, Giovanni Battista Migliori (1) ${ }^{4}$ \\ and Marieke J. van der Werf ${ }^{2}$
}

\begin{abstract}
Affiliations: ${ }^{1}$ Clinic-al Epidemiology and Medical Statistics Unit, Dept of Biomedical Sciences, University of Sassari - Research, Medical Education and Professional Development Unit, AOU Sassari, Sassari, Italy. ${ }^{2}$ European Centre for Disease Prevention and Control, Stockholm, Sweden. ${ }^{3}$ Dept of Pathophysiology and Transplantation, University of Milan, Cardio-thoracic Unit and Adult Cystic Fibrosis Center, Fondazione IRCCS Cà Granda Ospedale Maggiore Policlinico, Milan, Italy. ${ }^{4} \mathrm{WHO}$ Collaborating Centre for TB and Lung Diseases, Fondazione S. Maugeri, Care and Research Institute, Tradate, Italy.
\end{abstract}

Correspondence: Giovanni Battista Migliori, WHO Collaborating Centre for TB and Lung Diseases, Fondazione S. Maugeri, Care and Research Institute, via Roncaccio 16, 21049 Tradate, Italy.

E-mail: giovannibattista.miglioriafsm.it

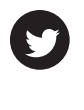

@ERSpublications

New European Union standards for TB care and prevention to achieve WHO target of TB elimination by 2050 in EU/EEA http://ow.ly/NlhE302UXLV

Tuberculosis (TB) is one of the most important clinical and public health infectious disease threats globally [1]. Its burden is substantial, with annually 9.6 million incident cases and 1.5 million deaths worldwide [1]. Since 1990, the previous decreasing global annual incidence trend has reversed due to the emergence of HIV/AIDS and the increase in TB-HIV co-infection. In addition, multidrug-resistant TB (MDR-TB) has emerged as a problem [1]. This change in the epidemiology has significantly challenged the control of TB through the World Health Organization (WHO) DOTS (directly observed treatment, short course) strategy, and forced international and national public health authorities to design and plan new operational strategies.

The current strong global determination to end the TB epidemic has prompted the WHO to completely revise its public health strategy and to prepare a comprehensive approach named the End TB strategy [2]. The long-term targets (i.e. for 2030 and 2035) and strategic pillars aim for a radical change to the current situation. The pillars include a strong focus on integrated patient centred care and prevention using modern, evidence-based methods, in addition to "bold policies and supportive systems" and "intensified research and innovation".

Integrated patient centred care and prevention should be supported by clinical and public health guidelines and standards for the management of TB patients. Guidelines are prepared at national level using the guidance and recommendations of the WHO and other international organisations.

The first international standards, the International Standards for Tuberculosis Care (ISTC) [3], were developed by an international group of experts, the Tuberculosis Coalition for Technical Assistance, and were well received by the global community working on TB prevention and control [4]. The usefulness of the ISTC for routine clinical care was shown by the fact that there was widespread uptake and adoption of the standards for the diagnosis, treatment and prevention of TB disease and TB infection by healthcare

Received: July 222016 | Accepted: July 252016

Support statement: Support was provided by the European Centre for Disease Prevention and Control (ECDC) (grant number: ECD.5879). Funding information for this article has been deposited with the Open Funder Registry.

Conflict of interest: Disclosures can be found alongside this article at erj.ersjournals.com

Copyright OERS 2016 
workers [5]. Ongoing scientific progress resulted in updates to the standards and the release of new editions in 2009 and 2014, focusing on the new epidemiological issues and including new scientific developments (e.g. MDR-TB, TB/HIV co-infection and comorbidities) [6].

The ISTC, by definition, are international and focus on minimum standards which should be adopted and followed by healthcare workers located in different epidemiological settings (i.e. high-, middle- and low-income and TB incidence countries) all over the world. Differences in the epidemiological situation and economic/financial resources available may allow for different levels of care and, consequently, countries with more resources available could aim for higher clinical and public health standards. This rationale encouraged the European Centre for Disease Prevention and Control (ECDC) and the European Respiratory Society (ERS) to prepare the European Union Standards on Tuberculosis Care (ESTC). The ESTC are an adaptation of the second version of the ISTC to the European Union/European Economic Area (EU/EEA) setting [7]. In this setting, where the majority of the countries are high-income, as well as low TB incidence countries, standards considerably "higher" than those listed in the ISTC were considered feasible and necessary.

The scientific background supporting and providing the evidence base for the development of the ESTC included the results of a survey carried out in large TB reference centres in the EU/EEA [8, 9]. In this survey the clinical files of drug-susceptible and drug-resistant TB cases were assessed and attending physicians interviewed. The survey showed several shortcomings in the management of TB disease, both in the clinical and in the public health area. The main results of the survey highlighted errors in the dosing of anti-TB drugs and duration of treatment, in the care of TB/HIV co-infected patients (e.g. inadequate administration of antiretroviral drugs), in infection control measures (e.g. incomplete adoption of administrative and environmental measures, personal protective equipment), and in the management of individuals exposed to index cases and diagnosed with latent TB infection [10, 11].

For the development of the ESTC, the ECDC and the ERS established a process that included convening a panel of over 30 experts who took part in a Delphi process to define a list of standards, based on a review of the evidence. The structure of the ISTC was followed, and the ESTC document was finalised and published in the European Respiratory Journal (ERJ) in 2012 [4, 8].

In 2014 , the third edition of the ISTC was published [12]. In this edition the standards were updated using new information that had emerged; new approaches that became feasible; and recommendations from new WHO guidelines. The ECDC and the ERS performed a comparison of the ESTC with this third edition to identify differences and to evaluate if a revision of the ESTC was necessary [12]. The conclusion of this evaluation was that a revision of the ESTC would be appropriate in 2016.

We are now in 2016, and the ECDC and the ERS are planning the revision of the ESTC. Before embarking on the revision, a short survey was launched to assess whether the ESTC standards have been useful and effective in daily practice. The survey was carried out by the ERS and the ECDC and targeted ERS members (i.e. clinical and public health specialists) and ECDC contacts. Out of 1362 individuals approached, $341(25 \%)$ responded to the survey. The majority of respondents were clinicians $(241,71 \%)$ and public health specialists $(77,23 \%)$. The top three countries where respondents were located were Romania, Italy and Spain. More than half of the respondents reported they were involved in TB prevention and care on a daily basis. The results showed that 229 (67\%) were aware of the ESTC before the survey was administered. Survey participants learned about the ESTC mainly through the ERS publications (i.e. the ERJ and newsletter) (89\%). Over $90 \%$ of the respondents that knew about the ESTC declared that the ESTC provided useful information (figure 1) and the majority appreciated the document. Analysis of web statistics and a literature review indicated that the ESTC article had been widely disseminated, with over 13971 abstract views and 11213 downloads by readers from over 50 countries. The article was cited in 121 documents, mostly peer reviewed publications (91\%).

The feedback of the respondents provided through the survey underscored the necessity to update all the sections of the current ESTC document, i.e. the sections on standards for TB diagnosis, treatment, addressing HIV infection and other comorbid conditions, and public health and TB prevention. More room should be given to diagnosis (i.e. molecular techniques), and to treatment of difficult-to-treat patients, particularly those with drug-resistant TB (e.g. use of new drugs), TB/HIV co-infection (e.g. new HIV regimens) and comorbidities (e.g. diabetes mellitus). Also, the standards related to management of latent TB infection should be expanded. On the basis of the survey feedback, it appears that there is a clear need to disseminate the new updated document, with the help and support of (inter-)national scientific societies and governmental and nongovernmental organisations.

The first step in preparing for the revision of the ESTC has already been taken and included the identification of a panel of experts by the ERS in collaboration with the ERS Respiratory Infections 


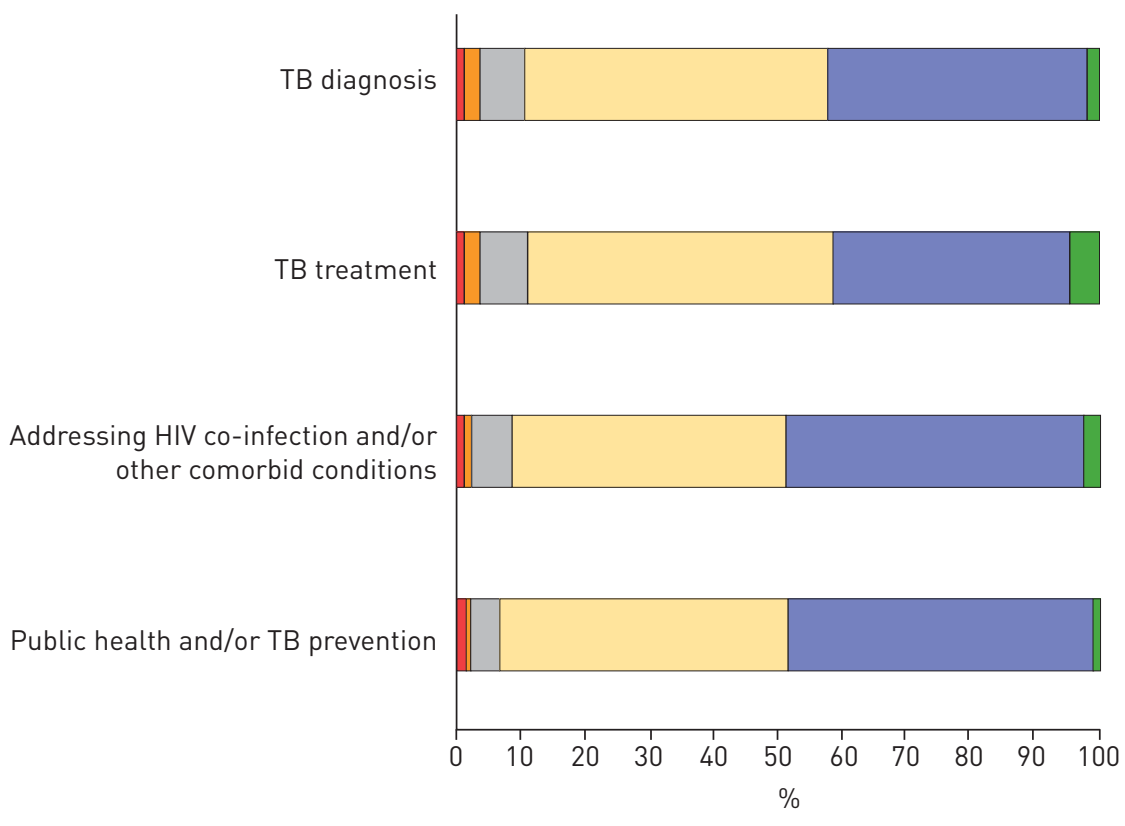

Not useful at all $\square$ Not very useful $\quad \square$ Neutral $\quad \square$ Useful $\quad \square$ Very useful $\quad \square$ No opinion

FIGURE 1 Responses to survey question "Do you feel that the European Union Standards on Tuberculosis Care provides useful information for...?" TB: tuberculosis.

Assembly. This panel will have a key role in the development of ESTC version 2, which will be an adapted version of the ISTC version 3 published in 2014. The ERS will coordinate the revision of the clinical part of the ESTC and the ECDC will lead the revision of the public health part.

In preparation of the revision of the ESTC, the ERS and ECDC will repeat the on-site evaluation of current clinical management, following the same methodology adopted in the past [7]. The findings of this new field survey will help the experts in the panel to better target the EU standards and to address shortcomings in TB care that need attention.

The End TB strategy targets of an $80 \%$ reduction in TB incidence and $90 \%$ reduction in TB deaths by 2030 should be achievable within the target deadline in EU/EEA countries although this will require an acceleration of the current rate of reduction in TB incidence in the EU/EEA [13, 14]. This requires implementing appropriate clinical and public health interventions using a patient centred approach, based on the latest evidence available $[15,16]$. The ESTC aims to be an easy-to-use tool that highlights what should be done to reach these targets and the revised ESTC aims to be a valuable document for clinicians and public health experts [17].

\section{References}

1 World Health Organization. Global tuberculosis report 2015. Document WHO/HTM/TB/2015.22. Geneva, WHO Press, 2015

2 Lönnroth K, Migliori GB, Abubakar I, et al. Towards tuberculosis elimination: an action framework for low-incidence countries. Eur Respir J 2015; 45: 928-952.

3 Tuberculosis Coalition for Technical Assistance. International Standards for Tuberculosis Care (ISTC). The Hague, Tuberculosis Coalition for Technical Assistance, 2006.

4 Tuberculosis Coalition for Technical Assistance. International Standards for Tuberculosis Care (ISTC). 2nd Edn. The Hague, Tuberculosis Coalition for Technical Assistance, 2009.

5 Pai M, Daley P, Hopewell PC. International standards for tuberculosis care: relevance and implications for laboratory professionals. Indian J Med Microbiol 2007; 25: 89-92.

6 Fair E, Hopewell PC, Pai M. International Standards for Tuberculosis Care: revisiting the cornerstones of tuberculosis care and control. Expert Rev Anti Infect Ther 2007; 5: 61-65.

7 Chakaya J, Raviglione M. Quality tuberculosis care. All should adopt the new international standards for tuberculosis care. Ann Am Thorac Soc 2014; 11: 397-398.

8 Migliori GB, Zellweger JP, Abubakar I, et al. European Union standards for tuberculosis care. Eur Respir J 2012; 39: 807-819.

9 Migliori GB, Sotgiu G, Blasi F, et al. Towards the development of EU/EEA standards for tuberculosis care (ESTC). Eur Respir J 2011; 38: 493-495.

10 Migliori GB, Sotgiu G, D'Ambrosio L, et al. TB and MDR/XDR-TB in European Union and European Economic Area countries: managed or mismanaged? Eur Respir J 2012; 39: 619-625. 
11 Sotgiu G, D'Ambrosio L, Centis R, et al. TB and M/XDR-TB infection control in European TB reference centres: the Achilles' heel? Eur Respir J 2011; 38: 1221-1223.

12 TB CARE I. International Standards for Tuberculosis Care. 3rd Edn. The Hague, TB CARE I, 2014. www.tbcare1. org/publications/toolbox/tools/access/ISTC_3rdEd_final_inter.pdf

13 van der Werf MJ, Sandgren A, D'Ambrosio L, et al. The European Union standards for tuberculosis care: do they need an update? Eur Respir J 2014; 43: 933-942.

14 Sotgiu G, Migliori GB. Is tuberculosis elimination a reality? Lancet Infect Dis 2014; 14: 364-365.

15 D'Ambrosio L, Dara M, Tadolini M, et al. Tuberculosis elimination: theory and practice in Europe. Eur Respir J 2014; 43: 1410-1420.

16 Getahun H, Matteelli A, Abubakar I, et al. Management of latent Mycobacterium tuberculosis infection: WHO guidelines for low tuberculosis burden countries. Eur Respir J 2015; 46: 1563-1576.

17 Pai M. Improving the quality of tuberculosis care: we need standards and strategies to translate them into practice. J Epidemiol Glob Health 2014; 4: 77-80. 